\title{
Heavy Metals Stimulate Human LINE-1 Retrotransposition
}

\author{
Shubha P. Kale ${ }^{1}$, Lakisha Moore ${ }^{1}$, Prescott L. Deininger ${ }^{2}$, and Astrid M. Roy-Engel ${ }^{2 *}$ \\ ${ }^{1}$ Dept. of Biology, Xavier University of Louisiana, 1 Drexel Dr. New Orleans, LA 70125, USA. \\ ${ }^{2}$ Tulane Cancer Center SL-66, Dept. of Epidemiology, Tulane University Health Sciences Center, 1430 Tulane Ave., New \\ Orleans, LA 70112, USA \\ *Correspondence to Dr. Astrid M. Engel. Email: aengel@tulane.edu
}

Received: 15 November 2004 / Accepted: 06 February 2005 / Published: 30 April 2005

\begin{abstract}
L1 and Alu elements are among the most active retroposons (mobile elements) in the human genome. Several human diseases, including certain forms of breast cancer and leukemia, are associated with L1 and Alu insertions in functionally important areas of the genome. We present data demonstrating that environmental pollutants, such as heavy metals, can stimulate L1 retrotransposition in a tissue culture system using two different types of assays. The response to these agents was equivalent when using a cell line with a stably integrated L1 vector (genomic) or a by introducing the L1 vector by transient transfection (episomal) of the cell. Reproducible results showed that mercury (HgS), cadmium (CdS), and nickel (NiO) increase the activity of L1 by an average of three (3) fold $p<0.001$. This observation is the first to link several carcinogenic agents with the increased retrotransposition activity of L1 as an alternate mechanism of generating genomic instability contributing to the process of carcinogenesis. Our results demonstrate that mobile element activation must be considered as one of the mechanisms when evaluating genomic damage/instability in response to environmental agents.
\end{abstract}

Keywords: L1; retrotransposition; cancer; genetic instability; heavy metals

\section{Introduction}

LINEs (LINE-1s or L1s) are long interspersed repeated elements with the capability of generating new copies that insert throughout the genome. LINE amplification has been highly successful through evolution contributing approximately $17 \%$ of the human genome [1]. In addition, it is believed that LINEs have also been responsible for the $11 \%$ of the genome made up of Alu elements [2]. L1 retrotransposition also can serve as a vehicle to mobilize non-L1 sequences such as exons or promoters into existing genes [3]. Overall, LINE activity has greatly contributed to the evolution of the human genome.

From a clinical view point, there are several reported examples of diseases caused by L1 insertions, including muscular dystrophy [4] and hemophilia A [5]. Mobility of L1 and Alu elements has also been shown to cause cancer, probably through somatic mutations [6, 7]. A variety of reports present data suggesting that increased activity of mobile elements may be involved in neoplastic progression [8]. For example, rodent LINE expression is increased in tumors $[9,10]$. In humans, L1 expression is increased in breast cancers [11] and testicular cancers [12]. Yet, to date, little is known about the environmental factors that can influence human retrotransposition. Until relatively recently, it was practically impossible to measure retrotransposition activity in mammals, particularly at the somatic level. There are a number of examples of stress-inducing factors that influence the expression and rate of transposition for an assortment of elements in various organisms [13 - 16]. Two reports evaluate different and environmental factors on L1 expression using an L1 promoter-luciferase assay, providing an initial indication that L1 may respond to external factors [17, 18]. However, this method only evaluates L1 promoter response in an artificial setting that can not accurately evaluate the retrotranspositional activity of an element. There is one report on the stimulation of Alu retrotransposition by genotoxic compounds, suggesting that these types of mobile elements can be influenced by the exposure of exogenous agents [15].

In 1996, the Kazazian laboratory designed a genetically marked L1 vector such that the selectable marker would be activated only following an RNA-mediated retrotransposition event [19]. This development immediately presented the application of this L1-vector in quantifying mammalian retrotransposition using tissue culture. We utilized this L1 insertion assay to create a model system and evaluate selected environmental pollutants, specifically heavy metals. Two approaches were utilized: one using a cell line with a stably integrated copy of the L1-vector that more accurately reflects the "natural" state of an L1 element, and the other using the transient introduction of the vector into the cells. We show that both approaches 
are useful in the evaluation of L1 activity after the exposure to environmental agents.

Metal pollutants pose a threat of toxicity to both humans and wildlife because of their wide distribution in the environment and workplace and their high persistence [20]. Humans get exposed to these heavy metals from numerous sources including contaminated air, water, soil and food. Exposure to heavy metals is not uncommon; in particular people who smoke, consistently expose their lungs to several of these metals. Reports indicate that epidemiological data predict that 1 to 18 lung cancer deaths/10,000 smokers may be attributed to inhaled cadmium in cigarette smoke [21]. Several heavy metals like cadmium and mercury are on the EPA's list of extremely hazardous substances hazardous chemicals:

[http://yosemite.epa.gov/oswer/ceppoehs.nsf/CAS].

Among their many hazardous effects, cadmium is recognized as a carcinogen and a teratogen, while mercury is classified as a neurotoxin (reviewed by [22]). Although both nickel and cadmium are classified as carcinogens, they are poor mutagens, suggesting an indirect mechanism of action [23, 24]. The precise mechanism in which these heavy metals induce carcinogenesis is undefined. In this manuscript, we report:

(1) Both stable and transient transfection assays are successful in measuring L1 retrotransposition in a reproducible and consistent manner.

(2) Mercury, nickel and cadmium significantly increase L1 retrotransposition in a dose-dependent manner.

(3) These stimulatory effects are not universal for all metals, as cobalt, zinc and magnesium had no stimulatory effect on the L1 retrotransposition.

This is the first report demonstrating the stimulatory effects of carcinogenic environmental pollutants on L1 retrotransposition. Our results also demonstrate the need to take into account DNA damage through mobile element activation as one of the mechanisms of action of genotoxic agents.

\section{Materials and Methods}

\section{Reagents}

The following compounds were purchased from SIGMA-Aldrich: cadmium sulfide, CdS 99.999\%, mercury (II) sulfide, HgS, 99.5+\% HgS and nickel oxide (NiO), 99.9\%. The filtered stock solutions of $\mathrm{HgS}$ and CdS were quantified professionally by AccuLab Inc. of Louisiana using method numbers 213.1 for CdS and 245.1 for $\mathrm{HgS}$ as described in USEPA Methods for Chemical Analysis of Water and Wastewater. The concentrations of the stock suspensions were determined to be $1.37 \mathrm{mg} / \mathrm{L}$ (ppm, parts per million) or $1370 \mathrm{ppb}$ (parts per billion) for $\mathrm{HgS}$ and $1.15 \mathrm{ppm}$ or $1150 \mathrm{ppb}$ for CdS. The doses tested in the assays were various dilutions (example from 50 fold to 3000 fold) of the stock solutions using regular DMEM medium lacking any antibiotics as a diluent. During any procedure (preparation or experimental), the suspensions were continuously shaken in the hood at low speeds to ensure the uniform suspension of the particles of CdS and HgS.

\section{Plasmids}

JM101/L1.3ACMV (a kind gift of Dr. John Moran) was used in the previously described retrotransposition assay [3]. The plasmid contains a full-length functional L1 element (schematic shown in Figure 1A). PIRES2EGFP (Clontech) contains a neomycin resistance expression cassette and was used in parallel in the retrotransposition assays as a combined control for transfection and cytotoxicity. All plasmid DNA was purified by alkaline lysis and twice purified by cesium chloride buoyant density centrifugation. Final evaluation of the DNA quality was performed from the visual assessment of ethidium bromide stained agarose gel electrophoresed aliquots.

\section{L1 Transient Transfection Assay}

HeLa cells (ATCC CCL2) were grown in a humidified, $5 \% \mathrm{CO}_{2}$ incubator at $37^{\circ} \mathrm{C}$ in Earl's minimal essential medium (EMEM). EMEM was supplemented with $10 \%$ fetal bovine serum. HeLa cells were seeded in T-75 flasks at a density of $1.5 \times 10^{5}$ cells/flask and grown for 20 hours prior to transfection. Cells were transfected with the Lipofectamine Plus (InVitrogen) for three hours using $1 \mu \mathrm{g}$ of either the $\mathrm{L} 1 \mathrm{or} 0.3 \mu \mathrm{g}$ the neomycin control plasmid with $18 \mu \mathrm{l}$ of the plus reagent and $12 \mu \mathrm{l}$ of Lipofectamine, following the manufacturer's protocol. The transfection mix was aspirated and replaced with the complete media supplemented with the appropriate dose of the compound evaluated. After a 48 hour period, the treatment was removed and the cells were grown for two weeks using selection media (containing $400 \mu \mathrm{g} / \mathrm{ml}$ G418) to obtain the neomycin resistant $\left(n e o^{\mathrm{R}}\right)$ colonies. Cell colonies were fixed and stained for 30 minutes with crystal violet $(0.2 \%$ crystal violet in $5 \%$ acetic acid and $2.5 \%$ isopropanol). A schematic of the time line is shown on Figure 3B.

\section{Generation of Stable L1-Vector Cell Lines}

L1-stable cell lines of NIH3T3 (ATCC CRL1658) with the integrated human L1 retrotransposition cassette were generated. To integrate the plasmid the method of electroporation was selected as it usually generates clones with the integration of only one copy of the plasmid. $10^{6}$ cells were electroporated with $6 \mu \mathrm{g}$ of JM101/L1.3 $\Delta \mathrm{CMV}$ using $300 \mathrm{~V}$ and $800 \mu \mathrm{F}$. The electroporated cells were grown under hygromycin selection to obtain colonies containing the integrated plasmid. A mouse cell line (NIH3T3) was utilized to facilitate the evaluation of the hygromycin resistant clones to detect the integrated plasmid that contains a human L1. Once the individual colonies were grown, they were subsequently evaluated for their L1 retrotransposition capability as determined by the generation of neomycin resistant $\left(\right.$ neo $\left.^{\mathrm{R}}\right)$ colonies. Two clones (3E and 28E) contained an integrated copy of the L1 vector and were also L1 retrotranspositionally competent. Clone 28E was utilized for most of the experimental evaluations, as it generated a low inherent background. 


\section{L1 Retroposition Assay Using Stable L1-Vector Clonal Cell Lines}

Stable L1-vector clonal cell lines (described above) were used to evaluate L1 retrotransposition. Each assay lasted for 10 weeks and consisted of two parts a) retrotransposition assay and b) parallel toxicity control:

(1) Testing the chemical for effects on retrotransposition: To keep the background retrotransposition rate to a minimum, a fluctuation analysis was carried out by first seeding 1-2 cells of clone 28E into 12-well plates. The cells were fed with DMEM medium containing 5\% FBS (Invitrogen) and $0.01 \%$ non-essential amino acids (Invitrogen), but lacking any antibiotics or antifungal agents. When the cells reached the 100cell stage, they were treated as described in the results with the heavy metal for two nonconsecutive 24 hour exposures (Figure 2C). Upon allowing the cells to recover for 24 hours, 250,000 cells were seeded per T75 flask and subjected to selection (G418 400 $\mu \mathrm{g} / \mathrm{ml}$ ) for 14 days. A schematic of the assay time line is shown on Figure 2C. As per the design of the L1 vector, only retrotransposed cells would be resistant to G418. The retrotransposed clones were stained with crystal violet for 3 hours, washed, allowed to dry and counted as raw data. For the fluctuation analysis, a minimum of twelve T75 flasks (each originating from a separate 1-2 cell seeding) were used for the determination of L1 retrotranspositional rate.

(2) Toxicity Control: To set up the toxicity control, two populations of NIH3T3 cells were utilized:

a. Native NIH3T3 cells lacking the L1 construct and sensitive to the antibiotic G418;

b. The neo ${ }^{\mathrm{R}}$ control cells, carrying an L1 insert and resistant to the antibiotic G418.

Both cell types were propagated from a 1-2 cell stage, and dosed at the 100-cell stage as in the experimental treatment. Then the cells were allowed to recover and divide until their numbers reached about one million $\left(10^{6}\right)$. However, at the stage of G418 selection, this protocol differed from the basic assay described above. Here, a mixture of 100 of the neo ${ }^{\mathrm{R}}$ cells plus 250,000 native cells were seeded into a T75 flask and grown under G418 selection for 14 days. The resultant clones were stained with crystal violet and counted and used to calculate the survival curve. The untreated group should generate an average of 100 colonies of $n e o^{\mathrm{R}}$ cells. If the chemical has a toxic effect on the cell viability, treated samples would present a lower number of retrotransposed clones than the expected 100. Finally, if the chemical has any effect on cell proliferation, the number of retrotransposed clones should be higher than the expected 100 in the dosed flasks.

\section{Statistical Analysis}

Analysis for the experiments using stable transfectants was performed using fluctuation analysis for mutations following the method originally described by Luria-Delbrück [25] with the appropriate modifications for the application to tissue culture assays [26]. Results obtained from transient transfection assays were evaluated using paired t-test.

\section{Results}

\section{Evaluation of L1 Retrotransposition}

About half a million L1 elements are found dispersed throughout the genome. However, most of them are 5' truncated [27] and estimates suggest that about 10003000 of them are full-length copies [28, 29] and only approximately $10 \%$ of those are likely to be active [30]. For our evaluation of the effects of L1 retrotranspositional activity, we designed an approach to parallel as closely as possible the conditions of the active L1 elements within the genome. The previously described L1 retrotransposition reporter vector, JM101/L1.3ACMV plasmid [19] was stably integrated into the genome. Individual clones were selected and amplified to homogeneity. The clones were evaluated for integration of the plasmid and for L1 retrotranspositional capability. The L1 vector used contains the sequence of an active L1 element driven by its endogenous promoter, and designed to allow expression of the neomycin resistance $\left(n e o^{\mathrm{R}}\right)$ gene only when the expressed L1 element goes through retrotransposition (schematic in Figure 1A).

The use of a L1-stable cell line allows for the evaluation of potential epigenetic factors, such as methylation, that can affect the activity of a genomic L1 element [31 - 33]. However, the integrated L1 vector is continually active, generating a low level of integrated copies that contain a functional neomycin resistance gene. Thus, the simple task of growing the cell line will generate a mixed population of neomycin sensitive (no new L1s) and neomycin resistant cells (Figure 1B). Not only is the population mixed, but the proportions change relative to when in the cell expansion period L1 integration occurs. For example, if we start with 10 cells and at this stage 1 insert occurs, this will translate in a $10 \%$ neo $^{\mathrm{R}}$ background. However, if the cells had divided and the L1 insert occurs at the 100-cell stage a $1 \%$ neo $^{\mathrm{R}}$ background is observed. In contrast, the transient transfection assay introduces the plasmid into the cells at the same time as the treatment, eliminating the background levels (Figure 1C). Thus, to properly evaluate effects of the treatment on the stable L1-cells, we performed the assay in a way to be able to apply fluctuation analysis (details in materials and methods). Basically, the assay is started with 1-2 cells and the cells are treated at the 100-cell stage, most of the initial pool of the 28-E cells will have a zero background for retrotransposition, although a few may have $1 \%$ or more background (one or more cells out of the 100).

At the end of the experiment it is very obvious which set of 100 had an initial background, as the number of clones will be at least an order of magnitude greater than expected. These points can be eliminated, leaving only the experiments that initiated with zero background. This approach is the basis of the 'fluctuation test' and its extended studies [25, 26, 34], originally designed to determine spontaneous mutation rates in cultured bacterial and mammalian cells. 

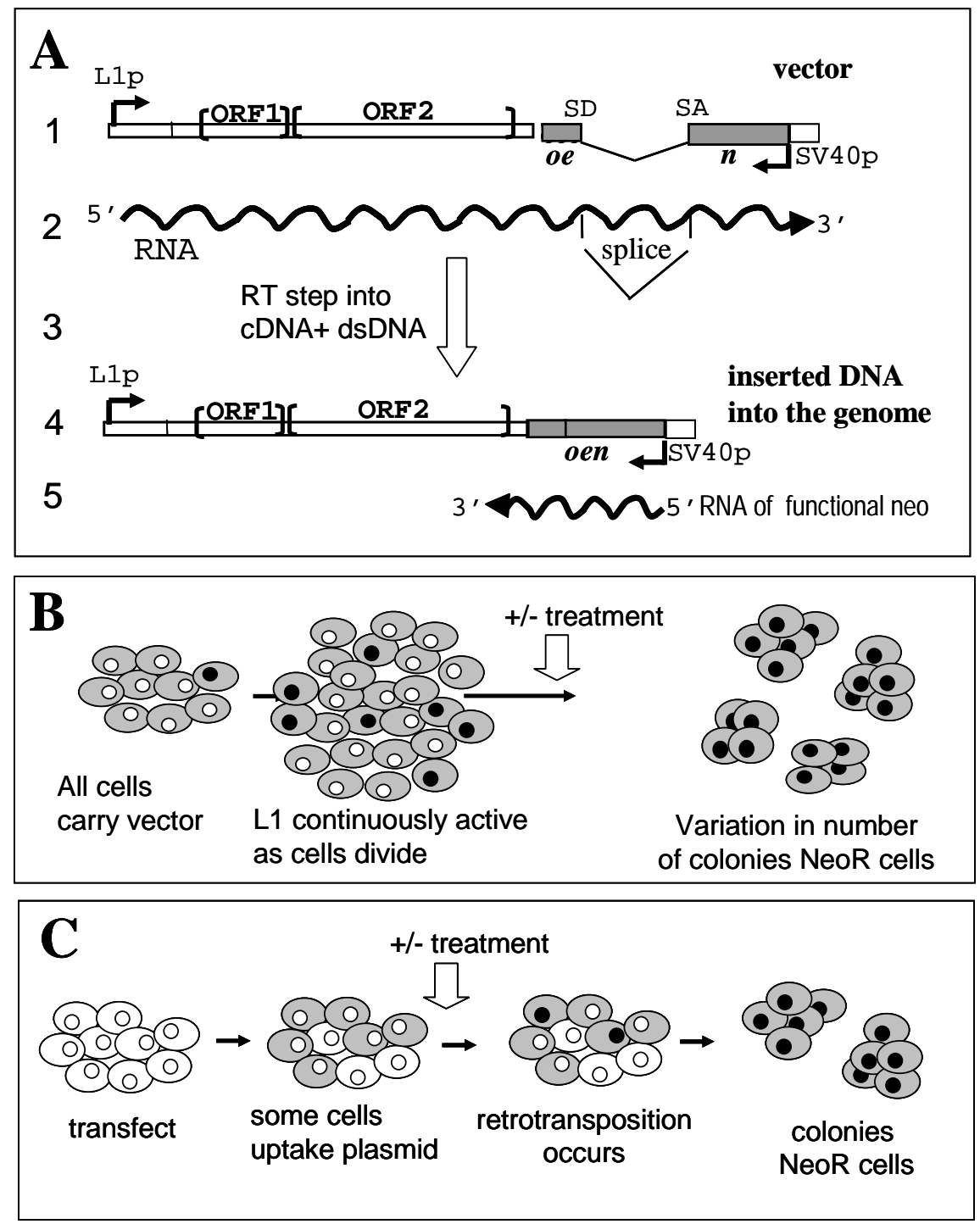

Figure 1: The L1 assay systems.

A. Schematic of the L1 vector. (1) The vector contains a full length L1 element with two open reading frames (ORF1 and ORF2). The construct contains the SV40 promoter (Sv40p) in the 3'UTR in the "reverse" direction that will transcribe a neo gene containing a "forward" intron that affects proper expression of the neomycin resistance. (2) RNA transcription is driven by the internal L1 promoter (L1p) located in the 5'untranslated region (UTR). The intron interrupting the neomycin resistance gene will be removed by splicing (SD: splice donor, SA: splice acceptor) only from RNA generated from the L1 promoter. (3) In the L1 retrotransposition process the RNA is reverse transcribed, followed by integration of the DNA into the genome. (4) The new L1 copy contains a functional neo gene. (5) Only newly integrated copies that retrotransposed from the spliced L1 RNA will generate neomycin resistance. The neo gene in opposite orientation relative to the L1 gene is shown as a gray box. RNA is represented by wavy lines with arrows to show direction of transcription. Note that the scale of the figure is not proportionally accurate.

B. L1 retrotransposition - L1-stable cell line assay. This assay is based on the use of a clonal cell line containing the L1 reporter vector integrated into the genome (gray cells). In these cells, the L1 cassette will have a baseline expression, which consistently generates new L1 inserts (gray with black nucleus). In other words, during the normal passage and seeding of the cells a steady background of neomycin resistant cells is being generated. Also, depending on when the event occurs (during the first division or later passage), the background level could represent from $1 \%$ up to $50 \%$ of the cells, even before the exposure to an agent. Thus, a fluctuation analysis is used to appropriately evaluate the effect of compounds in this assay.

C. L1 retrotransposition- transient assay. Cells are transiently transfected with the L1 reporter plasmid. Only a portion of the cells will uptake the plasmid and express the L1 RNA (gray cells). The cells are grown in the presence or absence of the compound tested (treatment). During this period a small fraction of the cells expressing L1, will generate a retrotransposition event as observed by the acquisition of neomycin resistance (gray cell with black nucleus). Growth under selection media will generate neomycin resistant $\left(n e o^{\mathrm{R}}\right)$ colonies each representing at least one retrotransposition event. 
A

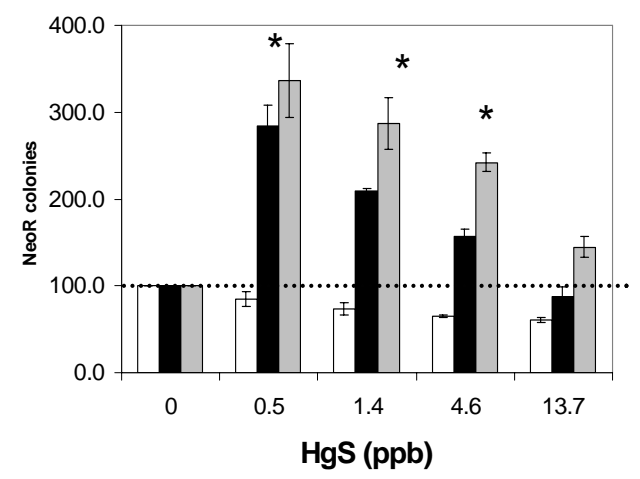

B
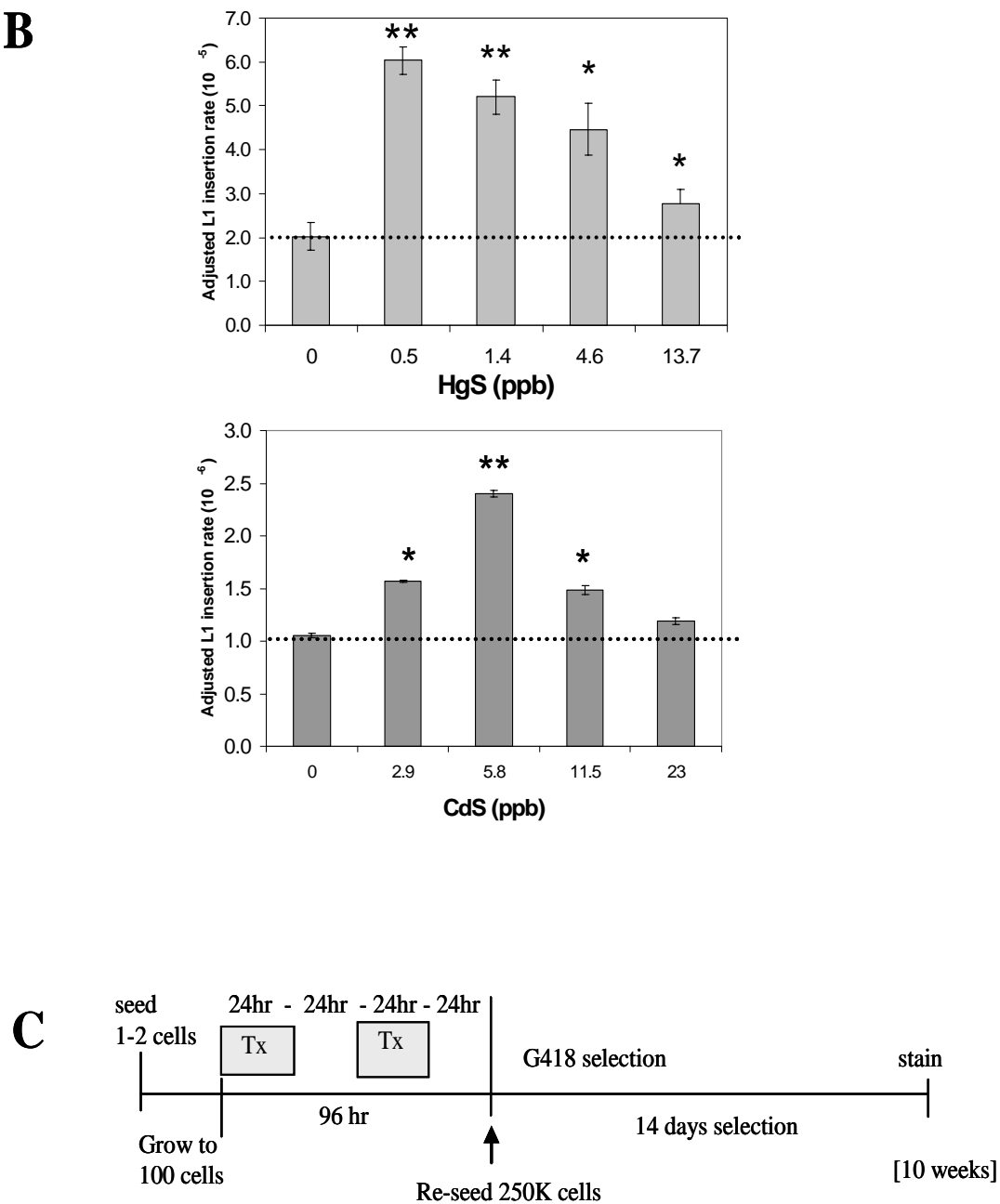

Figure 2: Effect of cadmium and mercury on L1 retrotransposition activity (L1-stable cell line assay).

A. $N e o^{\mathrm{R}}$ colonies from separate L1 transfections (black bar) treated with different doses of HgS are shown. The no treatment ( 0 dose) for each experiment was used as the $100 \%$. The average number of colonies from the toxicity control (white bar) was used to adjust the observed numbers (gray bar) as described in the text. Three independent assays in triplicate $(\mathrm{n}=9)$ were performed using clone $28 \mathrm{E}$ and error bars indicate one standard deviation. Statistically significant differences relative to the no treatment are indicated by asterisks [t-test $\mathrm{p}<0.01(*), \mathrm{p}<0.001(* *)]$.

B. L1 insertion rate: Fluctuation analysis was performed on the basic data (example for HgS shown in A) to obtain the adjusted L1 retrotransposition rate. Both CdS and HgS stimulate L1 retrotransposition from a genomically inserted L1-vector in a dose dependent manner with the maximum stimulus being around 3 fold. Average insertion rate is shown for $\mathrm{HgS}$ is $10^{-5}$ and CdS in $10^{-6}$ retrotransposition rate/cell division, with error bars representing one standard deviation. Statistically significant differences relative to the no treatment [t-test $\mathrm{p}<0.01(*), \mathrm{p}<0.001(* *)]$ are shown.

C. Schematic of time line of the L1-stable cell line assay: One or 2 cells of the L1 stable cell line were initially seeded and grown to 100 cell stage where they were exposed to the treatment with the selected agent. After treatment and recovery cells were reseeded (250,000 in a T75) and grown under selection for 2 weeks. $\mathrm{NeO}^{\mathrm{R}}$ colonies were counted after staining. The total duration of the assay is on average 10 weeks. 
A
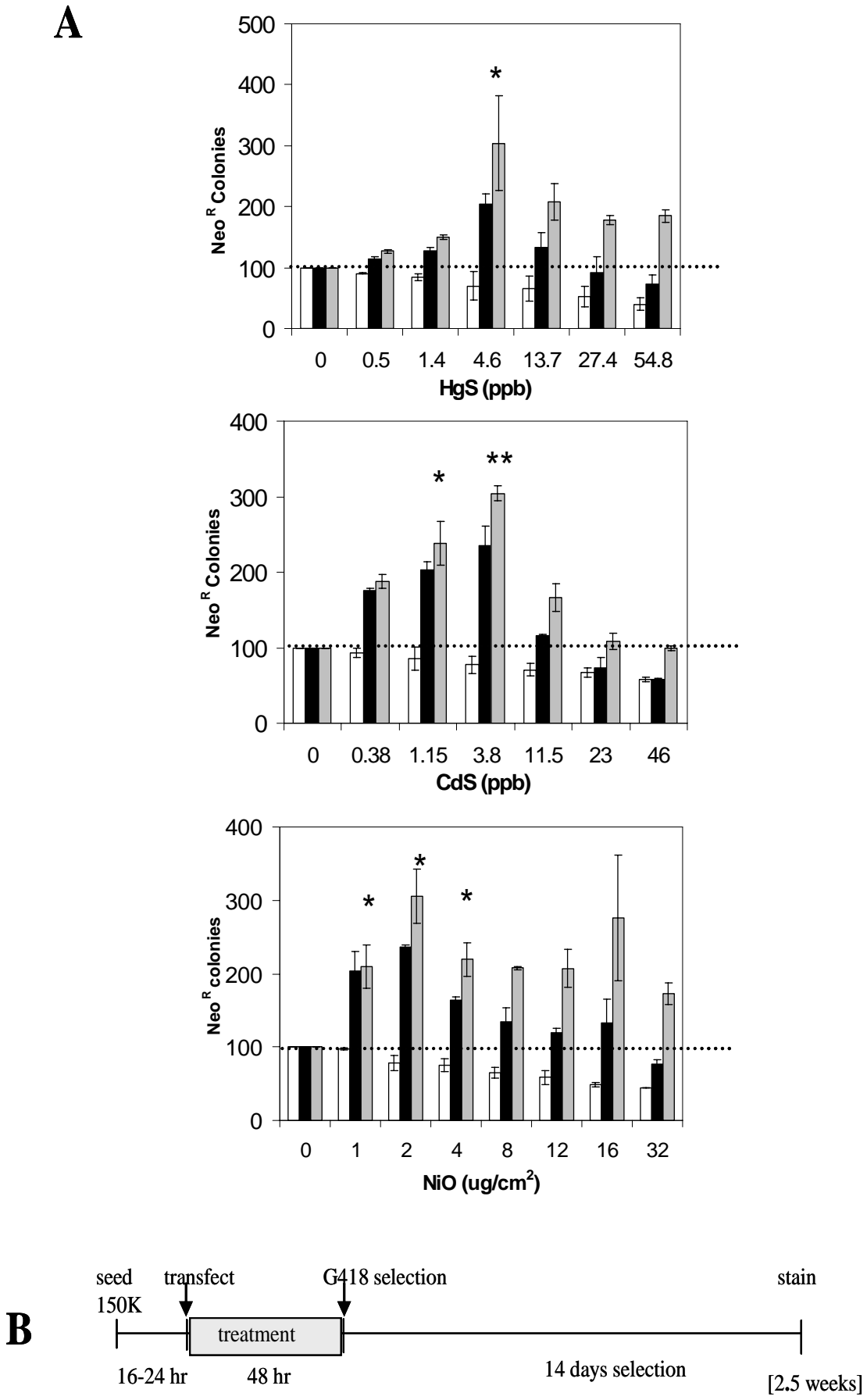

Figure 3: Effect of different compounds on L1 retrotransposition activity (transient transfection assay).

A. Metals stimulate L1 retrotransposition in a transient transfection assay: $N e o^{\mathrm{R}}$ colonies from separate L1 transfections (black bar) treated with different doses of HgS, CdS or NiO (X axis) are shown. An unrelated plasmid with neomycin resistance was used as a transfection and toxicity control (white bar). The no treatment (0 dose) for each experiment was used as the $100 \%$. In a similar manner as used for the L1-stable assay described in the text, the data were adjusted for toxicity (gray bar). Three independent assays in triplicate $(\mathrm{n}=9)$ were performed in HeLa cells and error bars indicate standard deviations. Statistically significant differences are indicated relative to the no treatment [ttest $\mathrm{p}<0.01(*), \mathrm{p}<0.001(* *)]$. All the metals tested with this assay show a stimulation of L1 retrotransposition comparable to that observed in the L1-stable assay.

B. Schematic of time line of the L1 transient assay: The L1-vector is transfected into cells that were seeded the previous day (150,000 in T75). Immediately after transfection (3 hour) cells are treated with the metal for 48 hours. Treatment is removed and cells are grown under selection for 2 weeks before staining. Total duration of the assay is 2.5 weeks. 
Heavy metals are cytotoxic to cultured cells, thus a toxicity control was included. A set of neo ${ }^{\mathrm{R}}$ cells and a set of native (non-L1 transfected) cells were treated in parallel to evaluate the influence of toxicity on cell survival and colony formation. The average number of colonies with no treatment was used as the $100 \%$ value for both the toxicity and L1 activity (Figure 2A). A correction factor was utilized to compensate for the toxic effects of the heavy metals when comparing the L1 retrotransposition to the control at different doses. For example, if dose $\mathrm{X}$ of $\mathrm{HgS}$ reduced the number $n e o^{\mathrm{R}}$ colonies of the control plasmid by $20 \%$, the number of colonies from the L1 with the same treatment was corrected to compensate for that $20 \%$ reduction (Figure 2A, gray columns). Using this approach, the plating control numbers were used to adjust the data obtained from the first part of the assay to determine the adjusted L1 retrotransposition rate and to obtain normalized results for plotting (Figure 2B).

\section{Heavy Metals Stimulate Retrotransposition from a Genomically Integrated L1}

We evaluated the water-insoluble carcinogenic forms (particulate) of the cadmium and mercury (CdS and HgS). Reports in the literature demonstrate that the phagocytosis of the particles allows for a more efficient internalization of high levels of these compounds into cells leading to a higher concentration of the compound within the cell [35 - 37]. Evaluation of mercury and cadmium demonstrated that both metals significantly increase L1 retrotransposition in a dosedependent manner (Figure 2). About a three-fold peak stimulus is observed on the number of $n e o^{\mathrm{R}}$ colonies recovered (Fig 2A) also reflected when L1 retrotransposition rate is calculated using fluctuation analysis (Fig 2B). As expected, at higher doses, the overall retrotransposition rate drops, probably due to toxicity leading to cell death. The toxicity control data were utilized to correct for toxicity (gray bar) as described above. The stimulation of retrotransposition by all the doses $(0.5,1.4,4.6$ and $13.7 \mathrm{ppb})$ of $\mathrm{HgS}$ and 2.9, 5.8, $11.5 \mathrm{ppb}$ of $\mathrm{CdS}$ were significantly different relative to the no dose control, $p<0.01$ (paired ttest).

\section{Heavy Metals L1 Stimulation Can Be Evaluated Using Transient Transfected Cells}

In this assay, HeLa cells were transiently transfected with the JM101/L1.3ACMV plasmid. The cells are then exposed to different doses of the different metals for 48 hours prior to the two weeks of G418 selection to obtain $n e o^{\mathrm{R}}$ colonies (Figure 3B). The no treatment (0 dose) was defined as $100 \%$ and the rest of the data were plotted relative to it. As toxicity control, an unrelated plasmid, containing a functional neo ${ }^{\mathrm{R}}$ gene (pIRES2EGFP) was transfected in parallel allowing for the evaluation of the influence of toxicity on both transfection efficiency and colony formation. Adjustment for toxicity was performed in the same manner described above. Our results demonstrate that both HgS and CdS generated a 3-fold peak stimulation of L1 activity equivalent to the stimulation observed in the stable L1 assay (Figure 3A). However, the dose range where the stimulation is observed varies between both assays. The L1-stable assay presents an increased sensitivity, seen as the detection of an effect with lower doses.

In addition, we evaluated other metals, and $\mathrm{NiO}$ also increased L1 activity (Figure 3), however soluble cobalt, magnesium and zinc chloride had no effect on L1 activity (data not shown).

\section{Discussion}

The mechanism by which some heavy metals induce cancer is unclear [22, 38 - 41]. The reactions of cells to environmental exposure are very complex, involving multiple pathways and cellular components. In this manuscript we present data supporting yet another mechanism by which heavy metals may cause disease.

Most of the steps involved in the L1 retrotransposition are currently poorly understood, making the determination of the mechanism by which these compounds induce L1 activity difficult. However, not all heavy metals evaluated have such stimulatory effects, so our results suggest that nickel, cadmium and mercury likely have a specific mechanism that result in these effects. The influence of these metals on the rate of L1 retrotransposition may occur at any of the retrotransposition steps, such as transcription or insertion into the genomic sites. We are currently evaluating the potential mechanism by which these metals may be affecting L1 retrotransposition (El-Sawy, et al. unpublished).

Until recently, it was impossible to measure the changes in the frequency of L1 jumping. Under the optimized conditions, both assays show reproducible, overlapping patterns of stimulatory effects of $\mathrm{HgS}$ and CdS (the cancerous forms) on L1 retrotransposition. The assay using a stably-integrated L1 vector better reflects the L1 elements, which are naturally present throughout the genome. There are extensive data suggesting that chromatin-level regulatory events may be important with L1 elements [31 - 33], which the L1-stable assay would more appropriately evaluate. However, the assay is highly complex, and demands a long time for completion ( $\sim 10$ weeks). As an alternative, we implemented the use of transiently transfected cells for the evaluation of different compounds on L1 activity. We realize that our transient assay does not fully mimic the natural L1 amplification. Because the L1 reporter system involves expression from a transiently transfected plasmid, the assay should not be influenced by factors such as methylation or chromatin structure. However, this alternative assay proved to be simpler, shorter in duration, highly reproducible and yielded comparable results to the stable-L1 vector assay. Although more evaluation is required, these assays may prove highly valuable in evaluating environmental compounds and their potential impact on genetic instability. Also, the assays used in this study are currently the only available that can assess the influence of environmental exposure on retrotransposon activity in humans.

Our study is the first of its kind to report stimulatory actions of certain heavy metals on human retrotransposition. Our findings that different compounds can stimulate retrotransposition are particularly relevant to the possible role of chronic exposures in both germline 
and somatic disease. Chronic exposure to heavy metals, and other toxicants, through workplace and environmental exposures may specifically increase the damage caused by retrotransposition over a long period of time. Previous data demonstrate that many heavy metals are not mutagenic by the standard bacterial assay systems; however, they are carcinogenic when tested in animal models [23, 24]. Further, our results demonstrate that even small amounts of these metals (in the range of $\mathrm{ppb}$ ) can cause on average about 3-fold increase in the rates of L1 retrotransposition. This is of high relevance when considering that the 'allowable' sewer-discharge limit for cadmium according to the official guidelines by the NEA (National Environmental Agency) is $1 \mathrm{ppm}$ (http://app.nea.gov.sg/cms/htdocs/article.asp?pid=1644), and that most humans do accumulate mercury through seafood ingestion and other sources in their lifetimes. Such cells, that have increased L1 retrotransposition due to exposure of CdS and HgS, very likely become more inclined to accumulate potentially deleterious mutations that may lead to human diseases like cancer. Accumulation of this damage may contribute to initiation and progression of cancer, as well as other diseases of chronic exposure. We present data to support a novel mechanism by which metals and other compounds can cause genomic damage through the modulation of mobile element activity. Our data suggest that the genetic damage caused by the stimulation of mobile elements present in the genome needs to be seriously considered as an alternate mechanism by which environmental/external compounds generate genetic instability and disease. Thus, we propose the following model of how the heavy metals cause genetic instability through the stimulation of mobile element activity.

\section{Model for Environmental Modulation of L1 Elements}

We present a model of the role the environment and mobile elements have on the generation of diseases and cancer (Figure 4). The integrity of the cellular homeostasis is constantly being assaulted by external components including environmental agents, such as oxidants, heavy metals, UV radiation, etc. The cell presents a complex system of regulatory and protective pathways to either correct (ie. DNA repair pathways) or prevent the continuation of an unrepaired event (cell cycle arrest and apoptosis). A "healthy" or disease free state is maintained as long as there is a balance of these external effects and the cellular controls. Mobile element activity is among the factors that influence this balance. For example, reports demonstrate that methylation of mobile elements maintains their expression under control [31, 42 - 43]. If the regulation by methylation is eliminated, the increase in expression of repeats leads cells to have an increased genetic instability with disastrous consequences [44]. Thus, external factors, such as heavy metals, affect the regulation of mobile element activity, allowing for an increase in altered cells. Mobile element activity may continue to be high in these cells, accumulating even more defects that in conjunction with inactivation of tumour suppressors, etc. may lead to a cancerous state.

The ability to directly or indirectly increase mobile element activity may represent a major contributory factor to genetic instability in somatic cells, leading to cancer initiation or progression, aging, or other diseases associated with chronic exposure to carcinogenic/toxic compounds. These data suggest that damage caused by retrotransposition may need to be considered when developing mechanistic models for genetic damage associated with environmental exposures.

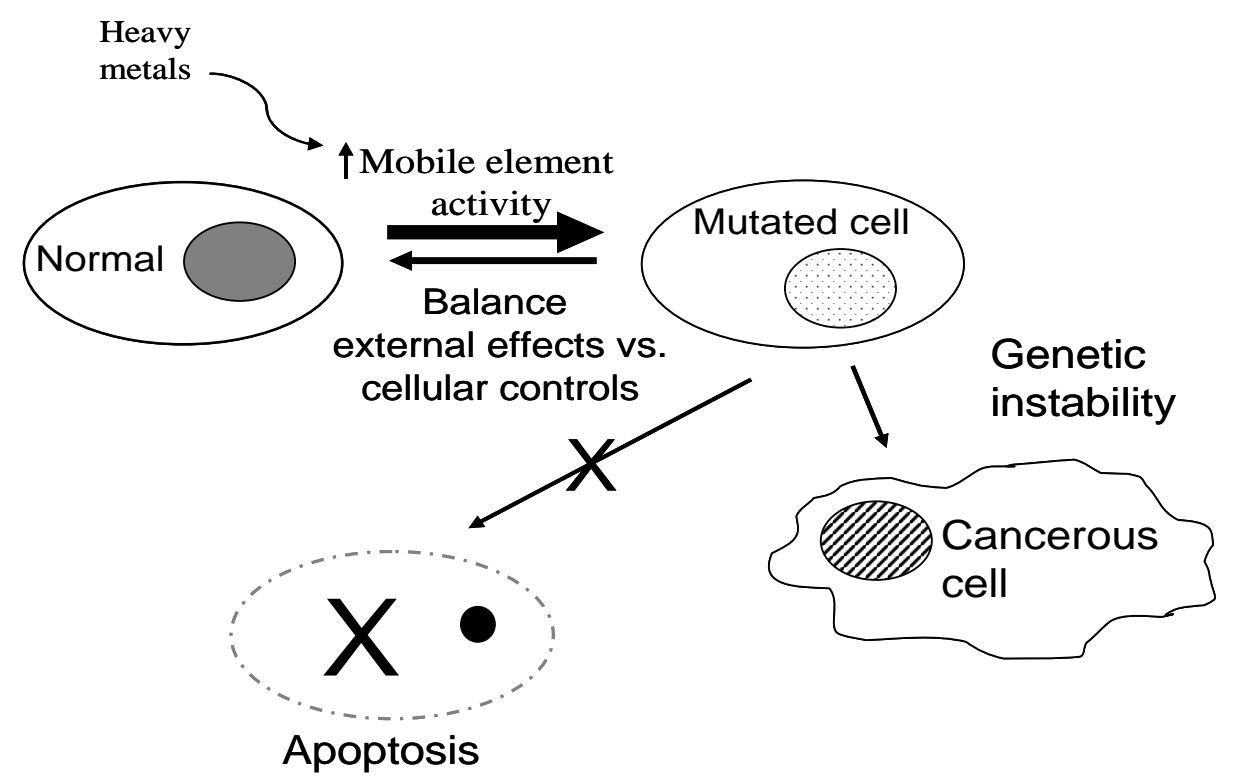

Figure 4: Proposed model for how metal stimulation of mobile elements may impact genetic stability.

The balance between normal cells and altered or mutated cells is depicted as an equilibrium. External and internal components can affect the outcome of this equilibrium. Normal cells are consistently being mutated or altered by external factors, such as UV light, etc. The damage generated can be either repaired or progress to two outcomes: (1) Apoptosis to eliminate the damaged cell or (2) Disease/cancerous cell. We propose that heavy metals shift the equilibrium through the increase of retroelement activity potentially leading to the accumulation of more mutated cells. 
Acknowledgements: This research was supported by National Institutes of Environmental and Health Sciences ARCH grant, 1S11ES09996 (SPK and PLD) NIH RO1 GM45668 (PLD), NSF EPS-034611 (PLD), the State of Louisiana Board of Reagents Support Fund (PLD) and NIH P20 RR020152 (AMR-E and PLD).

\section{References}

1. Smit, A. F.: The origin of interspersed repeats in the human genome. Curr. Opin. Genet. Dev., 1996, 6(6), 743-748.

2. Dewannieux, M.; Esnault, C; Heidmann, T.: LINEmediated retrotransposition of marked Alu sequences. Nat. Genet., 2003.

3. Moran, J. V.; DeBerardinis, R. J.; Kazazian Jr., H. H.: Exon shuffling by LI retrotransposition. Science, 1999, 283(5407), 1530-1534.

4. Narita, N.; Nishio, H.; Kitoh, Y.; Ishikawa, Y.; Ishikawa, Y.; Minami, R.; Nakamura, H.; Matsuo, M.: Insertion of a 5' truncated LI element into the 3' end of exon 44 of the dystrophin gene resulted in skipping of the exon during splicing in a case of Duchenne muscular dystrophy. J. Clin. Invest, 1993, 91(5), 1862-1867.

5. Kazazian, H. H.; Wong, C; Youssoufian, H.; Scott, A. F.; Phillips, D. G.; Antonarakis, S. E.: Haemophilia A resulting from de novo insertion of LI sequences represents a novel mechanism for mutation in man. Nature, 1988, 332, 164-166.

6. Deininger, P. L.; Batzer, M. A.: Alu repeats and human disease. Mol. Genet. Metab., 1999, 67(3), 183-193.

7. Kazazian, H. H. J.: Mobile elements and disease. Curr. Opin. Genet. Dev., 1998, 8(3), 343-350.

8. Hagan, C. R.; Rudin, C. M.: Mobile genetic element activation and genotoxic cancer therapy: potential clinical implications. Am. J. Pharmacogenomics., 2002, 2(1), 25-35.

9. Asch, B. B.; Asch, H. L.; Staler, D. L.; Anderson, G. R.: De-regulation of endogenous retrotransposons in mouse mammary carcinomas of diverse etiologies. Int. J. Cancer, 1993, 54(5), 813-819.

10. Grigoryan, M. S.; Kramerov, D. A.; Tulchinsky, E. M.; Revasova, E. S.; Lukanidin, E. M.: Activation of putative transposition intermediate formation in tumor cells. Embo J., 1985, 4(9), 2209-2215.

11. Bratthauer, G. L.; Cardiff, R. D.; Fanning, T. G.: Expression of LINE-1 retrotransposons in human breast cancer. Cancer, 1994, 73(9), 2333-2336.

12. Bratthauer, G. L. and Fanning, T. G.: Active LINE-1 retrotransposons in human testicular cancer. Oncogene, 1992, 7(3), 507-510.

13. Capy, P.; Gasperi, G.; Biemont, C; Bazin, C: Stress and transposable elements: co-evolution or useful parasites? Heredity, 2000, 85 (Pt 2), 101-106.

14. Fornace Jr, A. J.; Alamo Jr, L.; Hollander, M. C.; Lamoreaux, E.: Induction of heat shock protein transcripts and B2 transcripts by various stresses in Chinese hamster cells. Exp. Cell Res., 1989, 182(1), 1-74.

15. Hagan, C. R.; Sheffield, R. F.; Rudin, C. M.: Human Alu element retrotransposition induced by genotoxic stress. Nat. Genet., 2003.

16. Li, T.; Spearow, J.; Rubin, C. M.; Schmid, C. W.: Physiological stresses increase mouse short interspersed element (SINE) RNA expression in vivo. Gene, 1999, 239(2), 367-372.

17. Morales, J. F.; Snow, E. T.; Murnane, J. P.: Environmental factors affecting transcription of the human LI retrotransposon. I. Steroid hormone-like agents. Mutagenesis, 2002, 17(3), 193-200.

18. Morales, J. F.; Snow, E. T.; Murnane, J. P.: Environmental factors affecting transcription of the human LI retrotransposon. II. Stressors. Mutagenesis, 2003, 18(2), 151-158.

19. Moran, J. V.; Holmes, S. E.; Naas, T. P.; DeBerardinis, R. J.; Boeke, J. D.; Kazazian Jr, H. H..: High frequency retrotransposition in cultured mammalian cells. Cell, 1996, 87(5), 917-927.

20. Hirano, S. and Suzuki, K. T.: Exposure, metabolism, and toxicity of rare earths and related compounds. Environ. Health Perspect, 1996, 104 Suppl. 185-95.

21. Hertz-Picciotto, I. and Hu, S. W.: Contribution of cadmium in cigarettes to lung cancer: an evaluation of risk assessment methodologies. Arch. Environ. Health, 1994, 49(4), 297-302.

22. Ercal, N.; Gurer-Orhan, H.; Aykin-Burns, N.: Toxic metals and oxidative stress part I: mechanisms involved in metal-induced oxidative damage. Curr. Top. Med. Chem., 2001, 1(6), 529-539.

23. Chromium, nickel and welding. IARC Monogr Eval. Carcinog. Risks Hum, 1990, 491-648.

24. Meeting of the IARC working group on beryllium, cadmium, mercury and exposures in the glass manufacturing industry. Scand. J. Work Environ. Health, 1993, 19(5), 360-363.

25. Luria, S. E. and Delbrack, M.: Mutations of bacteria from virus sensitivity to virus resistance. Genetics, 1943,28491-511.

26. Capizzi, R. L. and Jameson, J. W.: A table for the estimation of the spontaneous mutation rate of cells in culture. Mutat. Res., 1973, 17(1), 147-148.

27. Sassaman, D. M.; Dombroski, B. A.; Moran, J. V.; Kimberland, M. L.; Naas, T. P.; DeBerardinis, R. J.; Gabriel, A.; Swergold, G. D.; Kazazian, H. H., Jr.: Many human LI elements are capable of retrotransposition. Nat. Genet, 1997, 16(1), 2,1 A3.

28. Adams, J. W.; Kaufman, R. E.; Kretschmer, P. J.; Harrison, M.; Nienhuis, A. W.: A family of long reiterated DNA sequences, one copy of which is next to the human beta globin gene. Nucleic Acids Res., 1980, 8(24), 6113-6128.

29. Grimaldi, G.; Skowronski, J.; Singer, M. F.: Defining the beginning and end of Kpnl family segments. Embo J., 1984, 3(8), 1753-1759.

30. Brouha, B.; Schustak, J.; Badge, R. M.; Lutz-Prigge, S.; Farley, A. H.; Moran, J. V.; Kazazian, H. H., Jr.: Hot Lls account for the bulk of retrotransposition in the human population. Proc. Natl. Acad. Sci. USA, 2003, 100(9), 5280-5285.

31. Yu, F.; Zingler, N.; Schumann, G.; Stratling, W. H.: Methyl-CpG-binding protein 2 represses LINE-1 expression and retrotransposition but not Alu transcription. Nucleic Acids Res., 2001, 29(21), 4493-4501. 
32. Takai, D.; Yagi, Y.; Habib, N.; Sugimura, T.; Ushijima, T.: Hypomethylation of LMEl retrotransposon in human hepatocellular carcinomas, but not in surrounding liver cirrhosis. Jpn. J. Clin. Oncol, 2000, 30(7), 306-309.

33. Hata, K.; Sakaki, Y.: Identification of critical CpG sites for repression of LI transcription by DNA methylation. Gene, 1997, 189(2), 227-234.

34. Lea, D. E.; Coulson, C. A.: The distribution of the numbers of mutants in bacterial populations. $J$. Genetics, 1949, 49, 264-285.

35. Cangul, H.; Broday, L.; Salnikow, K.; Sutherland, J.; Peng, W.; Zhang, Q.; Poltaratsky, V.; Yee, H.; Zoroddu, M. A.; Costa, M.: Molecular mechanisms of nickel carcinogenesis. Toxicol. Lett, 2002, 127(1-3), 69-75.

36. Singh, J.; Pritchard, D. E.; Carlisle, D. L.; Mclean, J. A.; Montaser, A.; Orenstein, J. M.; Patierno, S. R.: Internalization of carcinogenic lead chromate particles by cultured normal human lung epithelial cells: formation of intracellular lead-inclusion bodies and induction of apoptosis. Toxicol. Appl. Pharmacol, 1999, 161(3), 240-248.

37. Zelikoff, J. T.; Parsons, E.; Schlesinger, R. B.: Inhalation of particulate lead oxide disrupts pulmonary macrophage-mediated functions important for host defense and tumor surveillance in the lung. Environ. Res., 1993, 62(2), 207-222.

38. Coen, N.; Mothersill, C; Kadhim, M.; Wright, E. G.: Heavy metals of relevance to human health induce genomic instability. J. Pathol., 2001, 195(3), 293-299.
39. Hartwig, A.: Carcinogenicity of metal compounds: possible role of DNA repair inhibition. Toxicol Lett, 1998, 102-103, 235-239.

40. Kasprzak, K. S.: Possible role of oxidative damage in metal-induced carcinogenesis. Cancer Invest, 1995, 73(4), 411-430.

41. Tully, D. B.; Collins, B. J.; Overstreet, J. D.; Smith, C. S.; Dinse, G. E.; Mumtaz, M. M.; Chapin, R. E.: Effects of arsenic, cadmium, chromium, and lead on gene expression regulated by a battery of 13 different promoters in recombinant HepG2 cells. Toxicol. Appl. Pharmacol, 2000, 168(2), 79-90.

42. Gaudet, F.; Rideout, W. M., HI; Meissner, A.; Dausman, J.; Leonhardt, H.; Jaenisch, R.: Dnmtl expression in pre- and postimplantation embryogenesis and the maintenance of IAP silencing. Mol. Cell Biol, 2004, 24(4), 1640-1648.

43. Huang, J.; Fan, T.; Yan, Q.; Zhu, H.; Fox, S.; Issaq, H. J.; Best, L.; Gangi, L.; Munroe, D.; Muegge, K.: Lsh, an epigenetic guardian of repetitive elements. Nucleic Acids Res., 2004, 32(17), 5019-5028.

44. Bourc'his, D. and Bestor, T. R: Meiotic catastrophe and retrotransposon reactivation in male germ cells lacking Dnmt3L. Nature, 2004, 431(7004), 96-99.

Unpublished: El-Sawy, M.; Kale, S. P.; Dugan, C; Nguyen, T. Q.; Perepelitsa-Belancio, V.; Bruch, H.; RoyEngel, A. M.; Deininger, P. L: Nickel stimulates genetic instability through LI retrotransposition. 